\title{
SAGE Library
}

National Cancer Institute

\section{Source}

National Cancer Institute. SAGE Library. NCI Thesaurus. Code C16224.

Serial analysis of gene expression (SAGE) is novel method of assessing the variation in the type or amount of genes expressed in different tissues, at different times in development or in normal versus malignant cells. 